Instead of the Io0 $\mathrm{cc}$. burette mentioned above, one, two or three automatic burettes may be used; one constructed to automatically fill itself to $28.08 \mathrm{cc}$, , one to 42.12 , and one to $56.16 \mathrm{cc}$. These should be graduated to I/IO $\mathrm{cc}$. and the figures should run up the burette as in the one described above. Burettes of this kind can be obtained of Richards \& Co., of New York.

IABORATORY OF THE AGRICULTURAL EXPERIMENT STATION OF THE R. I. COLl. OF A. AND M. ARts.

A. W. BOSWORTH.

Note on Perkin's Test for Bicarbonates.-There was recently published, ${ }^{1}$ by F. M. Perkin, a test for bicarbonates based on the fact that bromine is liberated in a mixture of solutions of a bromide and sodium hypochlorite, if a dilute acid is added. Bicarbonates are sufficiently acid to bring about this reaction and may thus be distinguished from normal carbonates.

This test has proved very satisfactory in practice but the following exceptions must be noted: The test will not show the presence of ammonium bicarbonate nor can ammonium bromide be substituted for the sodium or potassium salts. Further than this, the presence of ammonium salts, even in very small quantities, prevents the reaction entirely. The ammonium salts experimented with were the chloride, carbonate, nitrate, sulphate, and oxalate, using solutions of the usual reagent strength and 5 per cent. solutions of sodium bicarbonate and potassium bromide.

For the test, I cc. each of the bromide and hypochlorite solutions were used and Io or I2 drops of the bicarbonate solution. Of the interfering ammonium salt solution, 2 drops, added before the bicarbonate, absolutely prevented the liberation of bromine, except in case of the oxalate, and a small additional quantity of this produced the same result.

Incidentally it may also be remarked that care must be taken that the hypochlorite solution used is reasonably fresh and has been kept away from light, or it will be sufficiently acid to lead to erroneous results.

Francis O. Taylor.

A Convenient Form of Table for Calculations of Chemicall Weights.-The author, having frequent occasion to check calculations of weights of substances made by students in quantitative analysis, has constructed a table, to enable him to quickly obtain

1 J. Soc. Chem. Ind., 22, 1375 (1902). 
such results. This is presented to the Section as an accessory in teaching which may prove instructive to the pupil and helpful to the teacher, and in a measure replace the use of the "rechentafeln" often so employed.

Upon coordinate paper, 500 by $400 \mathrm{~mm}$., the abscissa $500 \mathrm{~mm}$. is taken as 1000 , and there is laid off upon the vertical ordinate, the length corresponding to the ratio for reduction of weight sought for weight found. A straight line is then drawn from the origin to this point. Thus we take for the copper in cupric oxide 798 or for chlorine in silver chloride 247.

A diagonal line is thus drawn from the origin for each such ratio to be employed. We then read off upon the horizontal abscissa the measure corresponding to the figures of the weight found and the length of the ordinate at that point, cut off by the diagonal line for that substance, gives directly the amount of the body sought.

F. P. Dexington.

\section{NEW BOOKS.}

Princtiles of Inorganic Chemistry. By Harry C. Jones. New

York: The Macmillan Co. 1903. $x x+521$ pp. Price, $\$ 4.00$.

"The aim of this book is to add to the older generalizations those recently discovered, and to apply them to the phenomena of inorganic chemistry in such a way that they may form an integral part of the subject, and, at the same time, be intelligible to the student" (Preface).

The author states in the preface that the work is intended primarily for use by students of qualitative and quantitative analysis, and it may be presumed therefore that its aim is to modernize and expand the views of those who have already received some instruction in the elements of the science. At this stage, occasions for the application of modern ideas connected with ionization, mass action, solubility, and solution tension are certainly innumerable. Every operation in analysis, even the most trivial, bristles with opportunities for the use of these conceptions and can be performed intelligently and with unfailing success only by a student alive to the situation.

The book covers the general ground of a text-book of inorganic chemistry, but the theory of ionization and its application to the 